\title{
Experimental Investigation of Factors Influencing Operating Rotor Tip Clearance in Multistage Compressors
}

\author{
Reid A. Berdanier and Nicole L. Key \\ School of Mechanical Engineering, Purdue University, 500 Allison Road, West Lafayette, IN 47907, USA \\ Correspondence should be addressed to Reid A. Berdanier; rberdani@purdue.edu
}

Received 9 October 2014; Revised 3 January 2015; Accepted 4 January 2015

Academic Editor: Nekkanti Sitaram

Copyright ( 2015 R. A. Berdanier and N. L. Key. This is an open access article distributed under the Creative Commons Attribution License, which permits unrestricted use, distribution, and reproduction in any medium, provided the original work is properly cited.

\begin{abstract}
An analysis of compressor rotor tip clearance measurements using capacitance probe instrumentation is discussed for a three-stage axial compressor. Thermal variations and centrifugal effects related to rotational speed changes affect clearance heights relative to the assembled configuration. These two primary contributions to measured changes are discussed both independently and in combination. Emphasis is given to tip clearance changes due to changing loading condition and at several compressor operating speeds. Measurements show a tip clearance change approaching $0.1 \mathrm{~mm}(0.2 \%$ rotor span $)$ when comparing a near-choke operating condition to a near-stall operating condition for the third stage. Additional consideration is given to environmental contributions such as ambient temperature, for which changes in tip clearance height on the order of $0.05 \mathrm{~mm}$ ( $0.1 \%$ rotor span) were noted for temperature variations of $15^{\circ} \mathrm{C}$. Experimental compressor operating clearances are presented for several temperatures, operating speeds, and loading conditions, and comparisons are drawn between these measured variations and predicted changes under the same conditions.
\end{abstract}

\section{Introduction}

Rotor tip clearance height is known to be a significant contributor to overall compressor performance. In particular, previous studies have discussed its influence on total pressure rise, efficiency, and stall margin [1]. Current goals of increased efficiency and decreased fuel burn for gas turbine engines are focused toward more aggressive compressor designs featuring increased blade loadings and decreased blade heights in the rear block of high pressure engine cores. In these rear stages, blade aspect ratios are smaller, tip clearance heights are large relative to blade span, and endwall flows contribute to a more significant portion of the overall loss [2]. As a result, research focusing on the underlying flow physics for rotor tip leakage flows is growing in importance, particularly for tip clearance heights which are large as a percentage of overall blade span.

Ongoing research projects typically apply an extensive array of experimental techniques, but the ability to connect these experimental results to numerical models and validate computational tools is essential [3]. Although many computational fluid dynamics models struggle to accurately model the fundamental flow physics of tip clearance flows [4], the overall performance deltas due to clearance changes are typically comparable to experimental results. Small tip clearance changes and the related performance changes which occur as a result of changes to ambient temperature are typically considered negligible [5]. However, recent research has shown the measurability of these small performance changes in a multistage compressor [6]. As a result, it is imperative that the tip clearance heights are known for the conditions at which the experimental performance data are collected so that the compressor system can be appropriately modeled in numerical simulations.

Static rotor tip clearances-sometimes referred to as "cold" clearances-do not typically represent the operating, or "hot" running, clearances. In general, thermal growth, pressure forces, and circumferential forces due to high-speed blade rotation contribute to blade growth which leads to a clearance height change at different operating conditions and for different ambient conditions. Mechanical touch probes and erodible rub sticks offer low-cost options for measuring 
tip clearance during operation with easy implementation, but these methods only measure the tallest blade (i.e., the smallest clearance) during an entire test campaign [7], and no additional information is gained as a result of changes in operating condition or ambient temperature. Thus, noncontact clearance measurement systems, such as optical probes, eddy current sensors, or capacitive sensors, provide an alternative solution. Optical probes can be prone to failure if their line of sight is blocked by foreign objects, and thus the rugged design of capacitance sensors makes them a primary candidate for gas turbine applications [8].

The introduction of frequency modulated (FM) capacitance probe clearance measurement systems by Chivers [9] and Barranger [3] provided alternatives to earlier optical measurement systems. Continued component improvements have provided the ability to incorporate these high-accuracy systems in increasingly harsh environments. An overview of the advancement of capacitance probe measurements systems is given by Sheard [8].

Many authors have presented methods for modeling variations of tip clearances (e.g., Agarwal et al. [10], Kypuros and Melcher [11], and Dong et al. [12]). However, up to now, greater interest has been given to turbines, instead of compressors, due to the known benefits of active clearance control [13] and more significant thermal effects. Of the limited studies focused on predictive clearance modeling in compressors, Dong et al. discuss clearance changes due to loading condition at a fixed speed. Specifically, the authors noted changes of tip clearance approaching $0.5 \%$ span for the rear stages of a 10-stage compressor simulation. Further, simulations yielded misrepresentations of flow rate and efficiency when clearances corresponding to the static, or "cold," assembled configuration were implemented in the solution instead of estimated operating clearances.

While the work of Dong et al. [12] provides an important step in predictive modeling development for compressor tip clearances, there is a lack of experimental data in the open literature to support this model and advance the state of the art. Thus, the work presented here aims to fill that void by experimentally validating the variations of clearance with operating condition that was predicted by Dong et al. Further discussion is given to environmental effects on rotor tip clearances, and comparisons are drawn between the operating clearances measured by a capacitance probe system and the predictive clearances using the model proposed by Dong et al. Several recommendations are given for consideration in future test campaigns since experimental tip clearance studies which also measure the operating tip clearance are largely absent in the open literature.

\section{Experimental Methods}

This research was performed in the three-stage axial compressor research facility at Purdue University. Extensive experimental compressor performance data have been collected for an advanced tip clearance study implementing three different rotor tip clearance heights. In support of this project, a recent facility upgrade has incorporated a

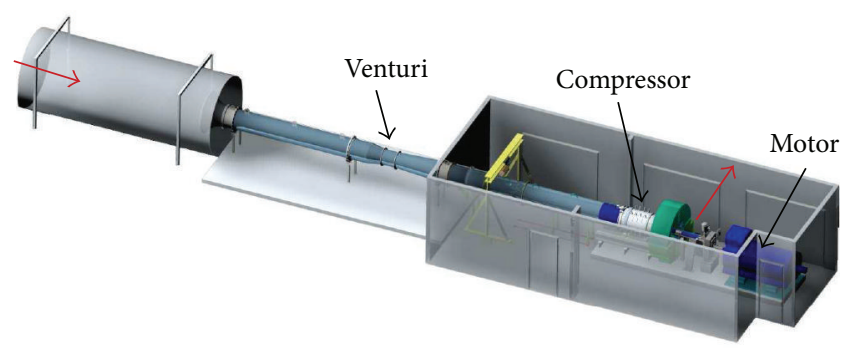

Figure 1: Purdue 3-stage axial compressor research facility.

capacitance-type tip clearance measurement system to measure the operating tip clearances in the compressor.

2.1. Compressor Facility. The Purdue three-stage axial compressor research facility is shown in Figure 1. The compressor draws unconditioned atmospheric air into a large settling chamber. A bell mouth directs the air into a long duct and through an ASME-standard long-form Venturi meter where the mass flow rate is measured. A nosecone directs the air into the $50.8 \mathrm{~mm}$ constant-annulus height (with a hub-totip ratio of 0.8333 throughout). Past the compressor exit, the air passes through a sliding-annulus throttle and is then directed out a scroll-type collector where the air exhausts to ambient conditions. The compressor is driven from the rear by a $1044 \mathrm{~kW}$ AC motor, and the driveline passes through a 5:1 speed-increasing gearbox to facilitate a corrected design speed of $5000 \mathrm{rpm}$. An encoder on the motor drive shaft and a proportional-integral-derivative control sequence maintain the rotational speed of the compressor within $0.01 \%$ of the desired set point. Aside from the motor control, an optical laser tachometer aimed at the high-speed shaft of the gearbox creates a transistor-transistor logic (TTL) signal which is used as a once-per-revolution (OPR) trigger for the high frequency response data acquisition systems.

The three-stage axial compressor features a 6061 aluminum casing and double-circular arc rotor blades made from 17-4 stainless steel. The rotor blade counts decrease through the compressor with 36 blades for Rotor 1, 33 blades for Rotor 2, and 30 blades for Rotor 3 . The stator vanes in this facility are all shrouded without hub clearances. Additional information related to the airfoil geometry can be found in [14]. The compressor casing was machined with tight tolerances to create the best possible vehicle for tip leakage flow measurements. Specifically, the inner diameter of the casing was specified as $609.6 \mathrm{~mm}+0.025 /-0.000 \mathrm{~mm}$ dimensionally, but additional geometric tolerances included a maximum $0.127 \mathrm{~mm}$ overall runout and a $0.051 \mathrm{~mm}$ surface profile shape. All dimensions were confirmed to be within tolerance using coordinate measuring machine (CMM) inspection techniques. Unless otherwise noted, the nominal rotor tip clearances for all data presented in this paper are $1.524 \mathrm{~mm}$, representing $3.0 \%$ of the annulus height. For reference, Table 1 provides the aspect ratio of the rotor blades and the corresponding tip clearances as a function of average blade 
TABLE 1: Rotor blade aspect ratios and clearance-to-chord values.

\begin{tabular}{lccc}
\hline & Rotor 1 & Rotor 2 & Rotor 3 \\
\hline Blade aspect ratio & 0.76 & 0.72 & 0.68 \\
Clearance-to-chord value (1.5\% span) & $1.14 \%$ & $1.08 \%$ & $1.02 \%$ \\
Clearance-to-chord value (3.0\% span) & $2.28 \%$ & $2.16 \%$ & $2.04 \%$ \\
\hline
\end{tabular}

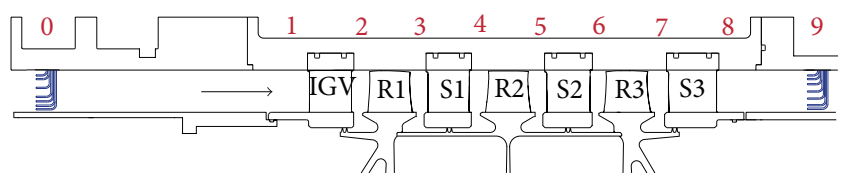

Figure 2: Compressor flow path and measurement plane locations.

chord for the two tip clearance configurations referenced in this document.

Steady pressure and temperature measurements throughout the compressor are standard for all operations. These data include a seven-element radial distribution of stagnation pressure and stagnation temperature at the inlet and exit planes of the compressor (locations 0 and 9 in Figure 2), a circumferential and axial distribution of static pressures at all axial positions 0 through 9 , and a series of surface-mounted T-type thermocouples on the outside of the compressor casing. These surface-mounted thermocouples are positioned at the aerodynamic interface plane (AIP) (plane 0 in Figure 2) and over each of the seven blade rows at a circumferential position of 124 degrees from the top of the compressor in the direction of rotor rotation, as shown in Figure 3. Past studies have shown that one surface temperature measurement is sufficient since circumferential surface temperature variations are less than $1^{\circ} \mathrm{C}$. For data sets requiring interstage flow information, seven-element rakes measuring stagnation pressure and stagnation temperature are also inserted at each of the axial positions labeled 1 through 8 in Figure 2. A careful design of the temperature measurement system has reduced the calculated uncertainty to less than $0.3^{\circ} \mathrm{C}$ for all channels, and the uncertainty of the measured absolute pressures is less than or equal to $50 \mathrm{~Pa}$.

2.2. Capacitance Probe System. The capacitance probe system utilized for this study is a CapaciSense 5-series FM clearance measurement system produced by Pentair Thermal Management. There are nine channels available, allowing three probes to be implemented for each of the three compressor rotors. The three probes are equally spaced circumferentially, 120 degrees apart, at positions of 105 degrees, 225 degrees, and 345 degrees-all measured from the top of the compressor in the direction of rotor rotation. These locations are also marked in Figure 3. Each of the nine probes was individually calibrated using a custom-designed calibration disk which represents the tip geometry of the rotor blade through scaling techniques proven by the manufacturer. The probes were all calibrated for operation from a rub condition $(0 \mathrm{~mm}$ clearance) to a maximum $5 \mathrm{~mm}$ clearance height.

The electronics chain for the FM tip clearance measurement system has several key components. The probes were

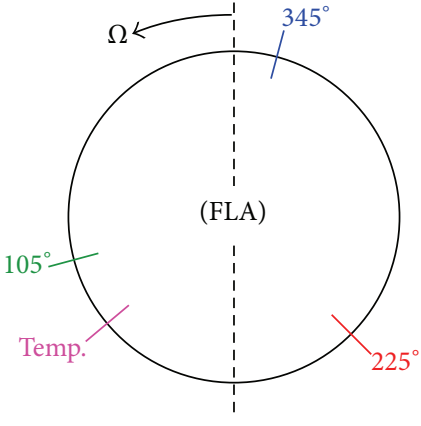

FIgURE 3: Circumferential instrumentation locations.

TABLE 2: Capacitance probe measurement system specifications.

\begin{tabular}{lc}
\hline Parameter & Value \\
\hline Operating frequency & $2 \mathrm{MHz}$ nominal \\
Oscillator sensitivity & $100 \mathrm{kHz}$ per pF \\
Demodulator sensitivity & $500 \mathrm{mV}$ per kHz typical \\
Measurement range & $5 \mathrm{~mm}$ calibrated \\
System resolution & $<0.001 \mathrm{~mm}$ (at $0.7 \mathrm{~mm}$ clearance) \\
Signal-to-noise ratio & $30-50$ typical \\
\hline
\end{tabular}

designed and built for application in this specific facility; a triaxial cable is permanently attached to the probe and provides enhanced rejection of electromagnetic interference. The use of a non-mineral-insulated cable limits the maximum operating temperature of this system to $260^{\circ} \mathrm{C}$. The oscillator drives the cable with an oscillating voltage (nominally $2 \mathrm{MHz}$ ). As the blade passes the probe, the measured capacitance modulates the driven frequency from the oscillator. This modulation is sensed by the carrier, and the demodulator converts the modulation frequency due to the blade passing event to a DC voltage. The DC voltage is correlated to a clearance height via the individual channel calibration. This proportionality between measured capacitance, frequency modulation, voltage, and tip clearance is the crux of the FM tip clearance measurement system.

The clearance measurement system is controlled from a set of "control and processing module" (CPM) computers. Each of these computers utilizes one Advantech PCI-1714UL data acquisition card capable of sampling at a rate of up to $10 \mathrm{MHz}$ per channel, as well as an external trigger which is linked to the OPR signal from the TTL tachometer signal. For the measurements presented in this study, all data were collected using the full $10 \mathrm{MHz}$ sampling capability of the data acquisition card. Other specifications for the capacitance probe measurement system are given in Table 2 .

The clearance calculation process provides a blade-byblade clearance output calculated from the peak-to-peak voltage for the typical blade pulse output signal (known as the blade passing signal (BPS)). As an alternative, the software applies a low-pass filter to the BPS output to create a DC voltage output signal which the manufacturer refers to as a "RMS" signal, although it should be noted that the low-pass filter mechanism does not represent a true root-mean-square 
TABLE 3: Representative primary uncertainty analysis components for capacitance probe measurement system.

\begin{tabular}{lc}
\hline Parameter & Uncertainty value \\
\hline System noise & $5 \mathrm{mV}$ \\
Oscillator temperature coefficient & $0.2 \%$ per ${ }^{\circ} \mathrm{C}$ \\
Demodulator temperature coefficient & $0.1 \%$ per ${ }^{\circ} \mathrm{C}$ \\
A/D card uncertainty & $1 \mathrm{mV}$ \\
Standard deviation of calibration & $80 \mathrm{mV}$ \\
Probe setback uncertainty & $0.0125 \mathrm{~mm}$ \\
\hline
\end{tabular}

calculation procedure. This RMS signal serves as a representative average measurement of the tip clearance for all blades by a particular probe. A separate calibration was performed for both the BPS and RMS output signals. All data presented in this study were calculated using data output from the RMS calculation procedure, but a comparison of the RMS with an arithmetic mean of BPS results (not shown here) agrees well.

\section{Uncertainty Analysis}

The manufacturer of the capacitance probe measurement system claims an umbrella uncertainty on the measurement system of less than $0.01 \mathrm{~mm}$. This value is based on historical comparisons with other measurement techniques, including laser measurements and rub sticks, as well as careful attention to the design, manufacture, and calibration processes to ensure minimal uncertainties. However, no formal uncertainty analysis had yet been performed to validate this claim, which represents more of a repeatability or comparability and does not consider uncertainty contributions from the electronics components.

Other authors have performed uncertainty analyses for capacitance probe measurement systems, including Satish et al. [15] and Müller et al. [16]. The calculations from Satish et al. are extremely thorough as a reference for overall uncertainty analysis, but the manufacturer of the system used for this study did not provide sufficient information to perform the calculations outlined by Satish et al. The uncertainty analysis included by Müller et al. was performed for a comparable FM capacitance probe system several years ago and therefore was used as a model for the calculations performed here. Table 3 outlines some of the representative contributing components of the uncertainty analysis for the electronics of the capacitance probe system used for this study. Using these components, a root-sum-squared (RSS) calculation technique was implemented to calculate the overall system uncertainty. Table 3 includes the temperature coefficients for the demodulator and oscillator, but these components were not considered in the uncertainty analysis for two reasons: (i) the demodulator is operated in a climatecontrolled environment to reduce thermal effects and (ii) the data presented in this paper were collected within $7^{\circ} \mathrm{C}$ of the calibration temperature, and convective cooling around the oscillators helps to reduce thermal effects related to temperature increases.
The uncertainty of the electronics system is ultimately a function of the nominal clearance measured by the probe since the standard deviation of the calibration data varied inversely with increasing clearances. By calculating the representative uncertainty at each calibrated nominal clearance, a curve representing calculated system uncertainty versus nominal clearance was created for each of the nine probes, as shown in Figure 4(a). Because the probe-to-probe variability in Figure 4(a) is small (less than $0.005 \mathrm{~mm}$ ) for nominal clearances of interest in this study (less than $2 \mathrm{~mm}$ ), a representative average of the nine probes was calculated and a 4 thorder polynomial fit was applied to those data, as shown in Figure 4(b).

To further quantify systematic errors, a typical compressor build may include measurement of the static tip clearance over each rotor at each of the nine tip clearance probe locations using a micrometer and a dial indicator, as described by Brossman [17]; these measurements are then compared with the clearances calculated from the capacitance probe system at a low-speed operation (200 rpm is a typical limit for low-speed measurement) at the start of a compressor measurement campaign. By measuring the operating clearances immediately after start-up and at a low operating speed, influences due to centrifugal effects and thermal gradients can be considered negligible.

\section{Results}

4.1. Rotational Speed. Figure 5 shows measured tip clearances as a function of the compressor operating speed during a typical startup process. These results are presented as a clearance difference with respect to the static clearance value:

$$
\Delta \tau=\tau-\tau_{\text {static }}
$$

In Figure 5, the compressor is accelerated through a series of discrete mechanical speeds to allow the oil temperature in the gearbox manifold to increase to an appropriate level. During this process, the effect of tip clearance variation due to the increasing speed is apparent. However, tip clearance changes due to thermal effects are minimal since the temperature rise through the compressor is low at part speed, and the overall acceleration time is relatively small. As a result, the measured clearance changes in Figure 5 are largely due to the centrifugal effects imposed on the rotor as the rotor blades grow with increased operating speed. A minor exception to this is somewhat apparent for compressor operating speeds above $4500 \mathrm{rpm}$, when the measured clearances increase slightly during constant mechanical speed operation. At these higherspeed "plateaus," small contributions due to temperature rise through the compressor are first noticeable. However, because the compressor operates at an open throttle condition (near-choke) during the startup process, the temperature rise remains very low. These changes in tip clearance with increasing rotational speed have been documented by other authors in the past $[8,9,16]$.

4.2. Loading Condition. Once a steady operating speed has been reached, the loading of the compressor can be increased 


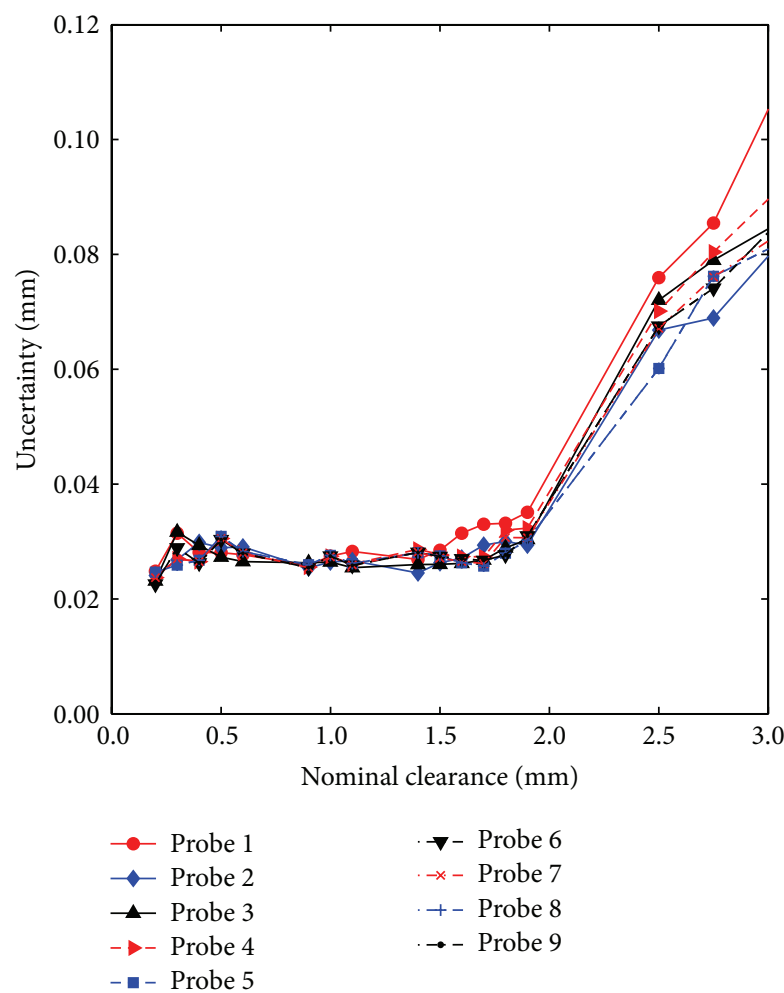

(a)

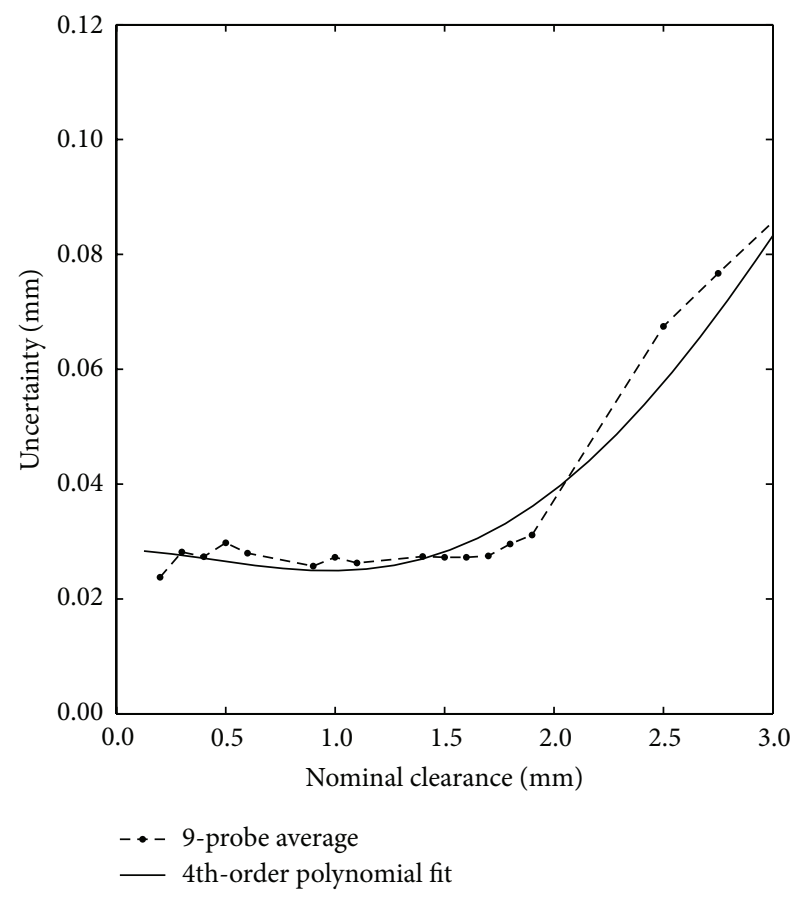

(b)

FIGURE 4: Uncertainty of tip clearance measurements. (a) All nine probes; (b) average.

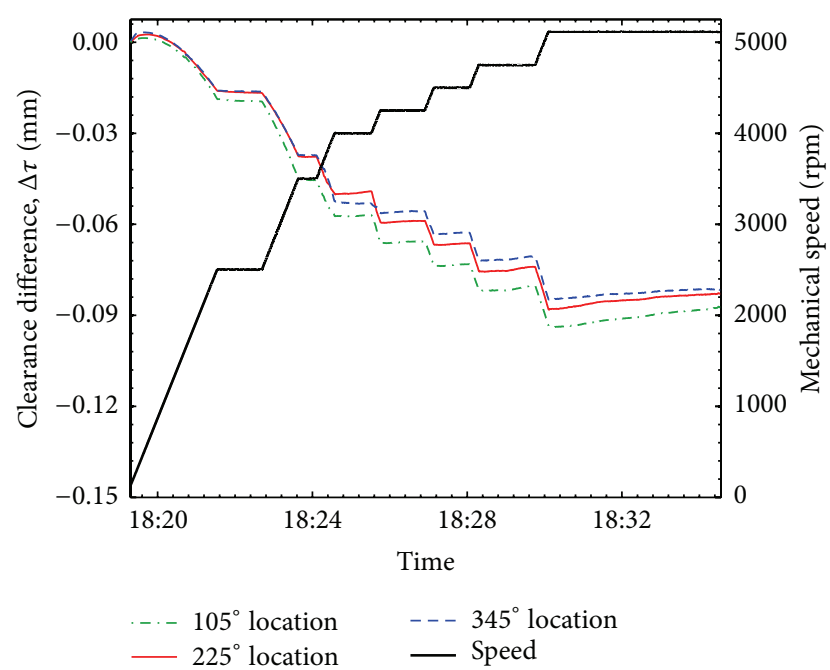

FIGURE 5: Clearance changes with speed for Rotor 2 during startup process at an open throttle operating condition.

in the direction toward stall by closing the sliding-annulus throttle at the exit of the machine. The overall compressor total pressure ratio is shown in Figure 6 at each of the four corrected operating speeds. In Figure 6, the total pressure is presented as a function of normalized inlet corrected mass flow rate, for which a normalized inlet corrected mass flow rate of unity denotes a nominal loading condition near the

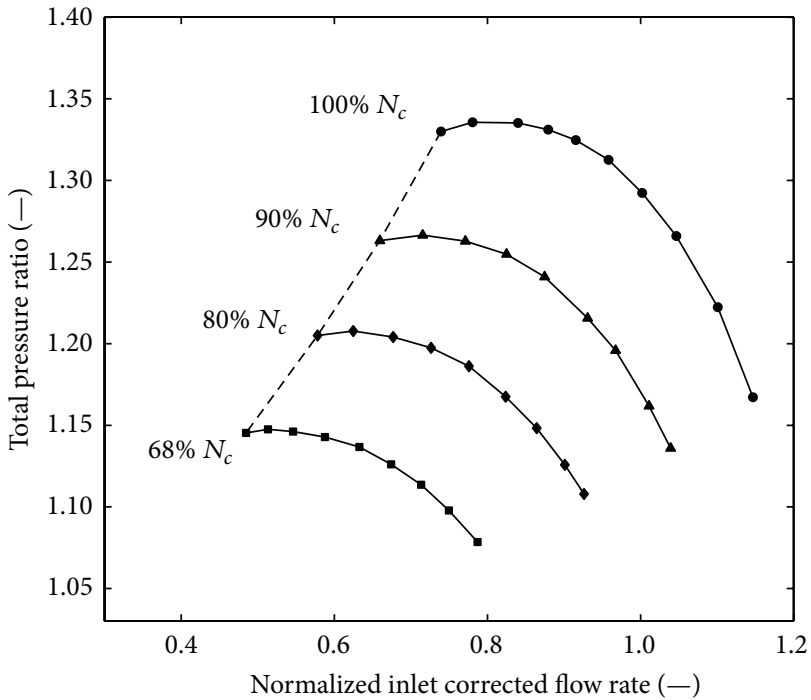

FIGURE 6: Overall compressor map at four operating speeds.

peak efficiency point. Each of the data points in Figure 6 represents a circumferential average of 20 vane positions with respect to measurement probes in order to remove variability due to stator wake effects. Each of the points in Figure 6 represents approximately one hour of compressor operating time. 

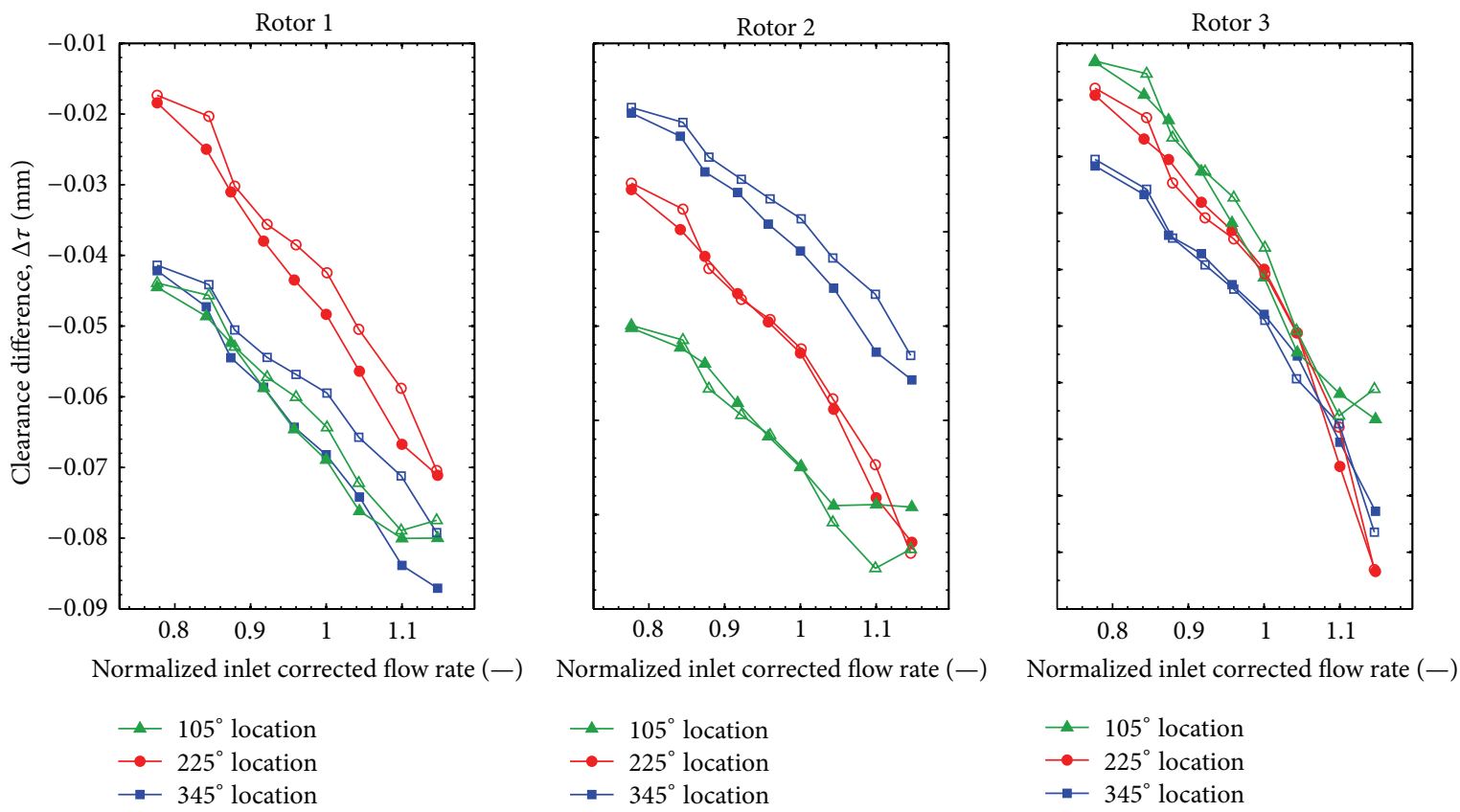

FIGURE 7: Clearance changes with compressor loading condition at a 100\% corrected operating speed.

Understandably, the temperature rise through the compressor increases with increased loading. As this occurs, the thermal effects begin to have a greater impact on the outer casing of the compressor. Specifically, moving from the open throttle position to a near-stall operating condition has the effect of increasing the stagnation temperature rise at the exit of each rotor blade row due to the increased work done on the flow.

Figure 7 shows the measured tip clearances for each of the nine loading conditions on the $100 \%$ corrected speedline from the compressor map in Figure 6. During the measurement of the overall compressor performance map in Figure 6, the operating clearances were measured, but since each data point in Figure 6 corresponds to approximately one hour of compressor operation, there is a possibility that any observed trends in measured clearance with loading condition are affected by variations in ambient temperature. In an effort to avoid false conclusions due to this potential thermal affect, the rotor clearances shown in Figure 7 were measured at the same loading conditions corresponding to the $100 \%$ corrected speedline from Figure 6. However, the data in Figure 7 were collected with approximately ten minutes between adjacent points during a period of time when the ambient temperature was relatively constant (within $2^{\circ} \mathrm{C}$ ). This time allows the machine to sufficiently reach a thermal equilibrium without allowing ambient temperatures to vary significantly. Furthermore, Figure 7 presents two series of measured clearances collected on separate occasions to represent the repeatability of the measurements as an alternative to the calculated electronic system uncertainty from Figure 4. With few exceptions, Figure 7 shows measurement repeatability of $0.01 \mathrm{~mm}$ or better, which is in agreement with the value reported by the system manufacturer.
The change in tip clearance with compressor loading condition has been predicted by Dong et al. [12] using a series of model equations for clearance change due to thermal and centrifugal effects. Predicted clearances calculated using this model were verified using experimental measurements collected from the General Electric $\mathrm{E}^{3}$ ten-stage compressor build. For a separate 11-stage compressor, Dong et al. predicted that increasing the loading condition from near choke to near-stall would affect the tip clearance by approximately $0.1 \%$ span for Rotor 4 but as much as $0.4 \%$ for Rotor 10 (due to an increase in temperature rise in the rear stages of the compressor).

The model presented by Dong et al. [12] utilizes a series of six calculated deformation contributions, each of which can be calculated using independent equations and combined to calculate the overall clearance. Specifically, the model calculates blade deformation due to thermal expansion and centrifugal forces, shroud deformation due to thermal expansion and pressure forces, and disc deformation due to thermal expansion and centrifugal forces. The six contributions are then combined with the static assembled clearance to determine the model clearance

$$
\begin{aligned}
\tau_{\text {model }}= & \tau_{\text {static }}-\left(u_{B, \text { thermal }}+u_{B, \text { centrif }}\right) \\
& +\left(u_{S, \text { thermal }}+u_{S \text {,pressure }}\right)-\left(u_{D \text {,thermal }}+u_{D, \text { centrif }}\right),
\end{aligned}
$$

where the subscripts $B, S$, and $D$ represent the blade, shroud, and disc, respectively. The equations representing these six contributing deformations have been applied using pressure and temperature data corresponding to the compressor performance points presented in Figure 6 for comparison with 

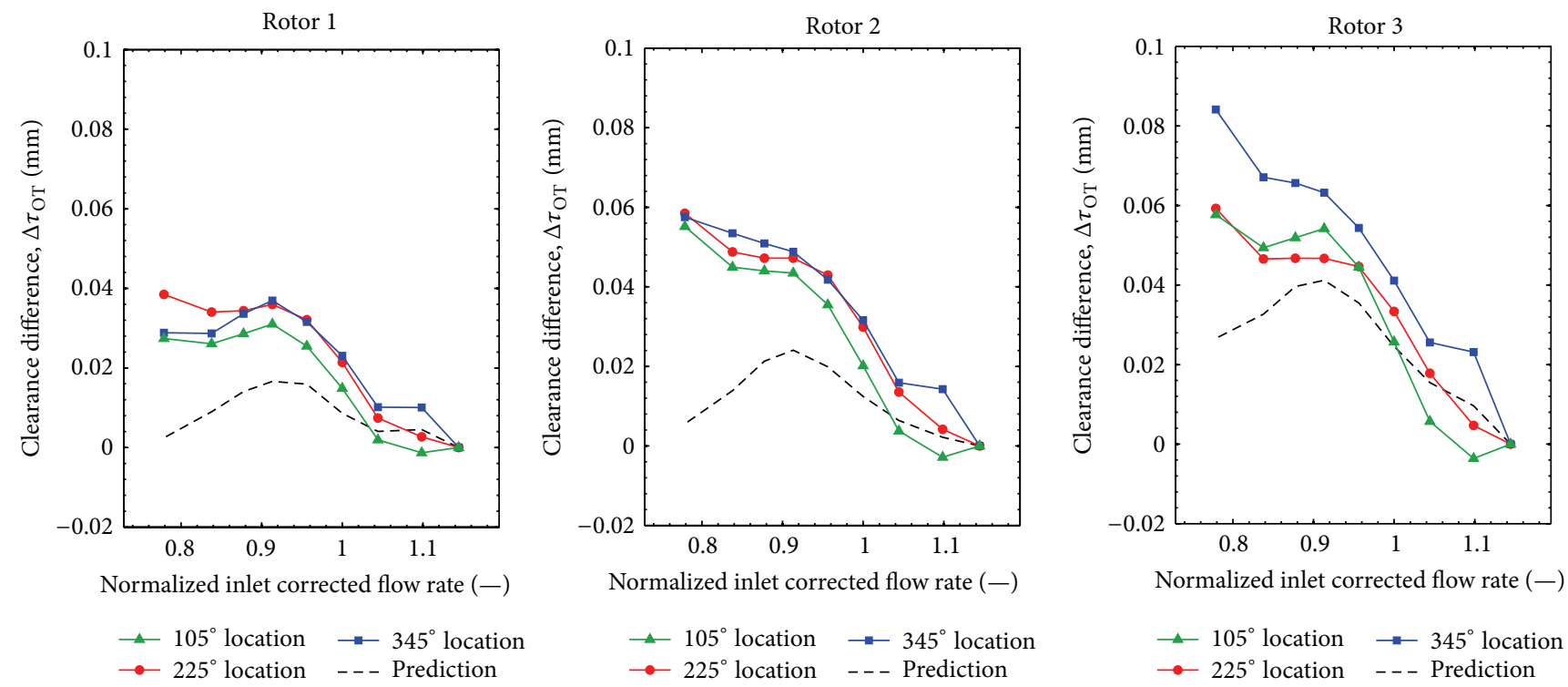

FIGURE 8: Predicted and measured clearance variations with loading condition, presented as a difference from the open throttle condition $\left(100 \% N_{c}\right)$.

operating rotor tip clearances measured simultaneously with the capacitance probe system.

The equations for the blade and shroud deformations were applied exactly as suggested by Dong et al. [12]. However, the equation for the disc deformation due to thermal effects was simplified to accommodate information available from the experimental results. Specifically, there were insufficient experimental data to allow calculation of the radial temperature distribution on the disc. As a result, the equations were simplified to approximate the disc temperature distribution by a constant temperature measured at the inner diameter of the flow path. This approximation is based on two primary pieces of information: (i) radial temperature gradients in the disc are expected to be small for the front stages of axial compressors and (ii) the integratedbladed-rotor (blisk) design of the Purdue three-stage axial compressor does not have air paths to facilitate convective cooling.

Using the series of stagnation pressures, stagnation temperatures, and static pressures in the flow path combined with temperatures measured on the outside of the casing, the six components required for the Dong et al. model were calculated. In the cases when static temperatures were required, the thermodynamic equation program REFPROP [18] was utilized in combination with measured parameters, allowing the application of high-accuracy equations to calculate thermodynamic properties including any pertinent humidity effects. Specifically, measured stagnation temperatures and pressures were combined with measured static pressure at the wall (assuming a constant radial distribution of static pressure due to a constant annulus height) to calculate the required static temperatures.

The results comparing the experimental data with the calculated model results are shown in Figures 8 and 9 for $100 \%$ and $90 \%$ corrected operating speeds. In this case, the results are presented as a difference with respect to the open throttle loading condition, denoted by the subscript OT:

$$
\Delta \tau_{\mathrm{OT}}=\tau-\tau_{\mathrm{OT}} .
$$

Considering first the results of the $100 \%$ corrected speedline data shown in Figure 8, there is a noticeable lack of agreement between the measured clearances and the predicted clearances for normalized inlet corrected mass flow rates less than one. Specifically, for the results from Rotor 2 in Figure 8, the near-stall point (lowest mass flow rate) shows a discrepancy nearly two times the uncertainty presented in Figure 4 and at least five times the repeatability shown in Figure 7 . On the other hand, the $90 \%$ corrected speedline data in Figure 9 show excellent agreement between the predicted and measured clearances. Similar agreement between predicted and measured clearances also exists for the $80 \%$ and $68 \%$ corrected speedlines (not shown here).

The model equation presented in (1) suggests that the discrepancy for the $100 \%$ corrected speedline results could come from an overprediction of blade and/or disk growth or an underprediction of shroud growth. To more closely consider the source of the errors, the individual components are presented in Figure 10. From this figure, the contributions to the predicted clearances are driven primarily by the thermal growth, which yield clearance differences several orders of magnitude larger than the pressure or centrifugal components.

4.3. Ambient Temperature Effects. Analysis of the data corresponding to the results in Figure 8 shows that a significant temporal variation of ambient temperature (on the order of $3^{\circ} \mathrm{C}$ ) exists for the data at low inlet corrected mass flow rates. These variations of temperature are present throughout the data collection period due to changes of ambient conditions. Of course, the corrected parameters defining the operating 

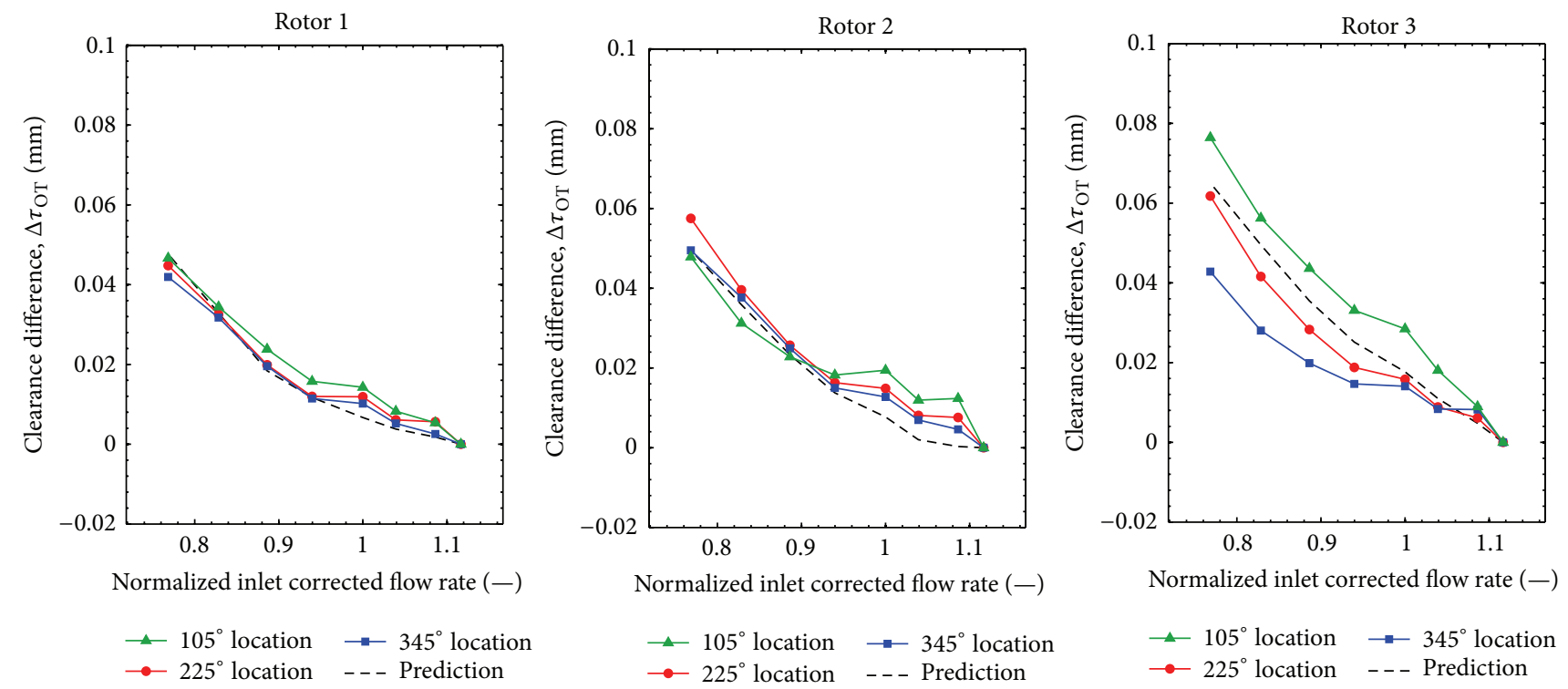

FIGURE 9: Predicted and measured clearance variations with loading condition, presented as a difference from the open throttle condition $\left(90 \% N_{c}\right)$.

point (rotational speed, mass flow rate) appropriately account for these varying ambient conditions in overall performance, but the averaged data which were utilized to calculate the temperatures required for use in (2) do not appropriately represent the required parameters. Thus, an adjustment was made to the prediction calculations such that the temperature utilized for the thermal components of (2) represents the temperatures measured at the exact same time as the measured tip clearances. Figure 11 shows that the adjustment does not remedy the entire discrepancy, but it more closely represents the measured values on the order of the uncertainty of the measurements. No adjustments were made to the nonthermal components of (2) due to their relatively small contribution shown in Figure 10.

Careful consideration is always given to ensure that a thermal equilibrium is reached prior to collecting these data. However, the data presented thus far suggest that the clearance variations with loading condition can also depend on the ambient conditions. Figure 12 shows this by comparison of two different tip clearance cases corresponding to different ambient conditions. Specifically, the data presented previously for the $3.0 \%$ clearance was susceptible to variations of ambient temperature for the low flow rates (as discussed above), whereas the $1.5 \%$ tip clearance results were collected over a period of time when the ambient temperature was nearly constant, which leads to the increased clearance growth at the low flow rates. The change of nominal tip clearance between the two cases poses a potential source of variability, particularly in terms of temperatures, but these data were collected during periods of similar weather and previous studies have shown that the temperature rise through the compressor is nearly identical for these two tip clearance cases.

To further emphasize the effect of ambient temperature on measured rotor tip clearance, Figure 13 shows the results of the $80 \%$ corrected speedline for one of the tip clearance configurations. It is important to note here that the lower rotational speed creates less temperature rise through the compressor. This lower temperature rise leads to clearance measurements which are less sensitive to loading condition. In other words, the measured and predicted clearances for adjacent operating points are nearly constant.

Considering the data in Figure 13, a stretch of severe weather forced a stop of the experimental campaign after the completion of the three points with the lowest flow rates, and the data collection process resumed on the following day. As shown in Figure 13, the discontinuity of ambient conditions for between the third and fourth points in the order of increasing flow rates caused a discernable discontinuity of rotor tip clearance (measured and predicted). This jump in clearance is approximately $0.06 \mathrm{~mm}$, which represents an $8 \%$ change of operating tip clearance for the nominal tip clearance of $1.5 \%$ span $(0.762 \mathrm{~mm})$, and is attributable to a change in ambient temperature of approximately $15^{\circ} \mathrm{C}$. Although there is no measureable change of performance parameters due to this difference of rotor tip clearance in this compressor, these results suggest that it is advisable to avoid a "stop and start" when a comparison of tip clearance information between adjacent data points is desired.

In these cases of varying ambient temperature and other environmental considerations, it is possible that the changing air composition could affect the dielectric properties enough to skew the capacitive effect, thereby artificially introducing the measured clearance changes shown here. Chivers [9] analyzed the relative permittivity of the dielectric material (for this study, air) at several temperatures and pressures representing a $220 \mathrm{kN}$ thrust class gas turbine engine. From that analysis, Chivers showed that increases in pressure and temperature due to flow through the compressor would affect the relative permittivity of air by approximately $0.05 \%$ 

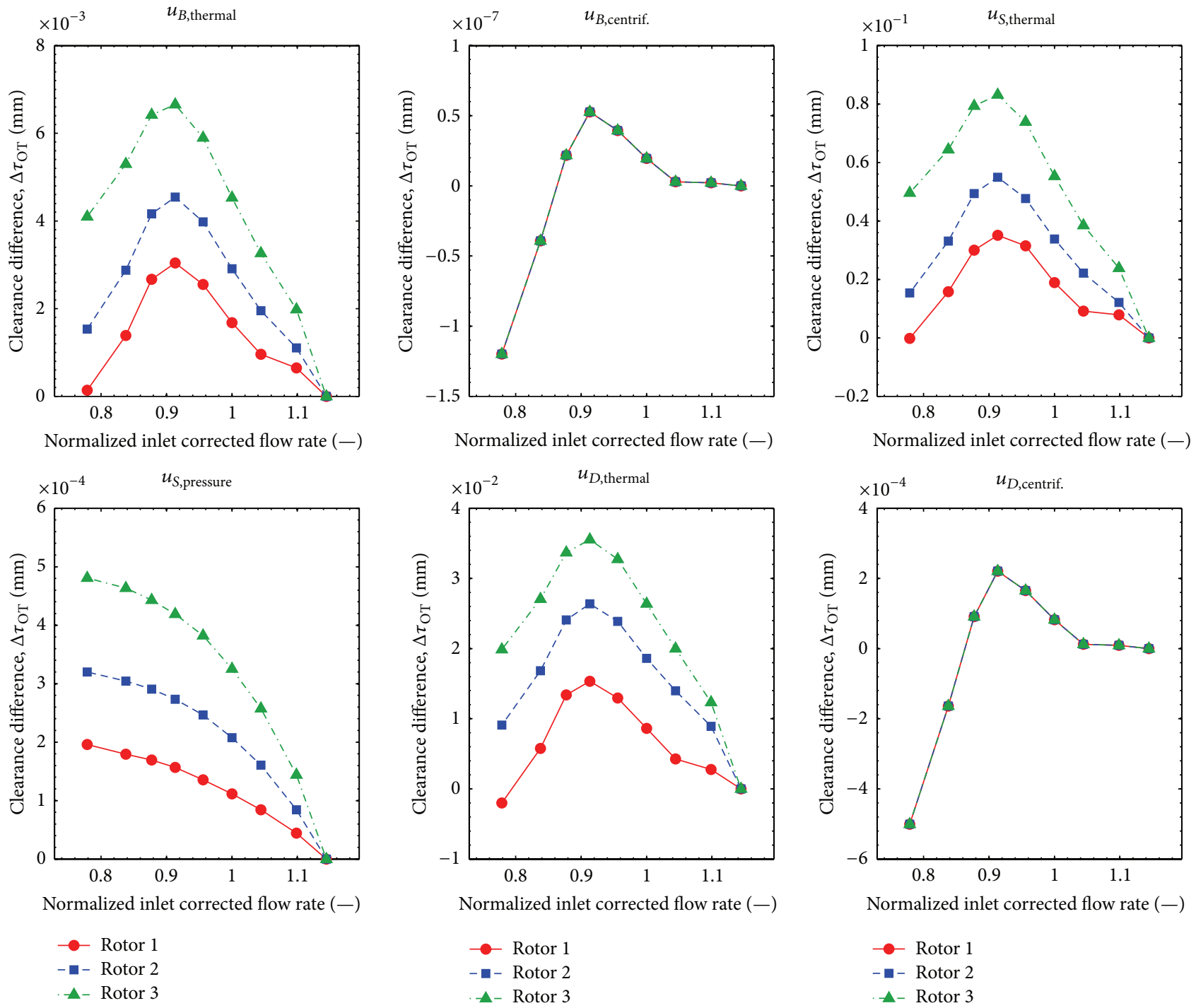

FIGURE 10: Components of predicted clearances from (2) corresponding to data for Figure 8.

compared to the same properties at the inlet of the machine. Further, Baxter [19] addresses the concern of humidity effects on relative permittivity. An increase of relative humidity from $40 \%$ to $90 \%$ at $20^{\circ} \mathrm{C}$ (a representative average for the data presented here) would affect the relative permittivity and, therefore, the measured capacitance on the order of $0.007 \%$ an order of magnitude less than changes due to temperature alone. From these values, it is expected that the changes of dielectric properties do not have a significant effect on the measured clearances.

4.4. Thermal Equilibrium Considerations. To this point, analysis of rotor tip clearance effects due to rotational speed, loading condition, and ambient temperature has been considered. Allusion has been given to thermal equilibrium, but primarily in the framework of ambient temperature variations due to time of day. Thus, the time associated with approaching a condition of equilibrium based on measured clearances was also considered explicitly in the context of thermal equilibrium.
The compressor was operated at a steady rotational speed of $2500 \mathrm{rpm}$ and was allowed to reach a state of equilibrium as determined from measured thermocouples and tip clearance measurements. At that point, the operating speed was increased to $5150 \mathrm{rpm}$ (a mechanical speed relating to a $100 \%$ corrected rotational speed based on ambient conditions) at a linear rate of $1100 \mathrm{rpm}$ per minute, and the corresponding response of measured clearances and shroud outer diameter temperatures was observed. In addition to the mechanical speed in Figure 14(d), the measured ambient temperature is also shown to verify that the measured temperature and clearance changes in Figures 14(a)-14(c) are not a result of changes in ambient temperature. These clearance results are shown in Figures 14(a)-14(c) as a difference with respect to the static clearance values.

Referring to Figure 14, immediately after the increase of speed, the measured clearances decrease in a nonlinear fashion. Throughout this speed change process, the surface 


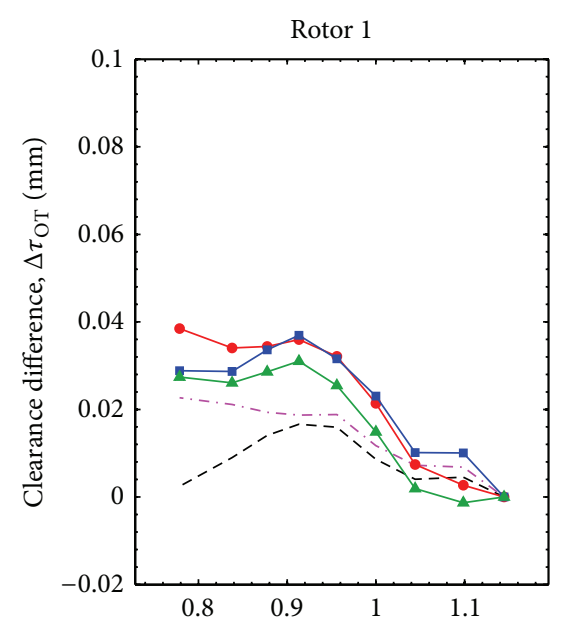

Normalized inlet corrected flow rate (-)

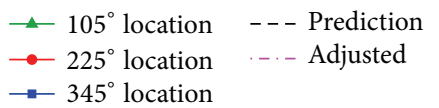

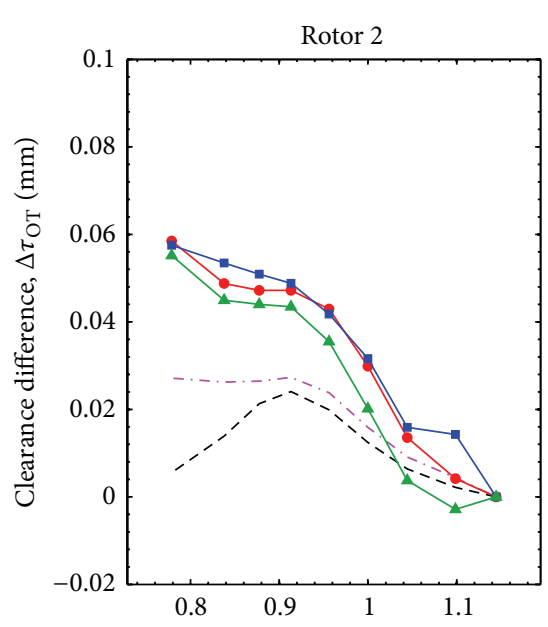

Normalized inlet corrected flow rate (-)

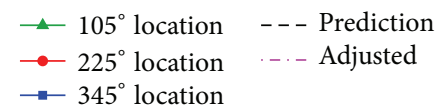

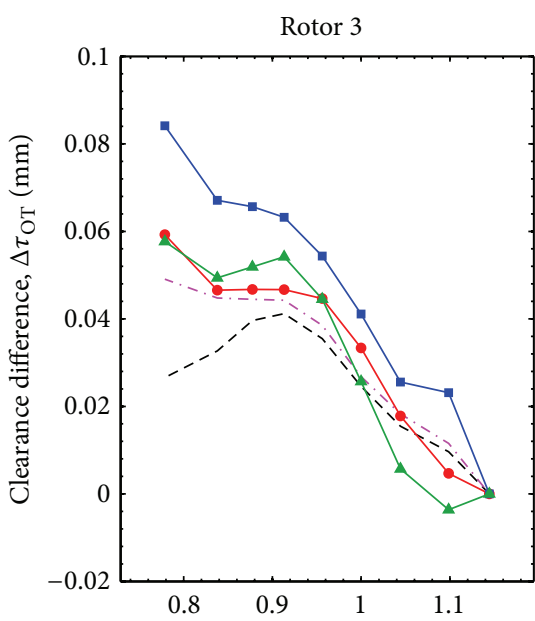

Normalized inlet corrected flow rate $(-)$

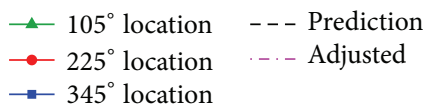

Figure 11: Predicted and measured clearance differences from the open throttle loading condition including a temperature adjustment for the predicted results $\left(100 \% N_{c}\right)$.
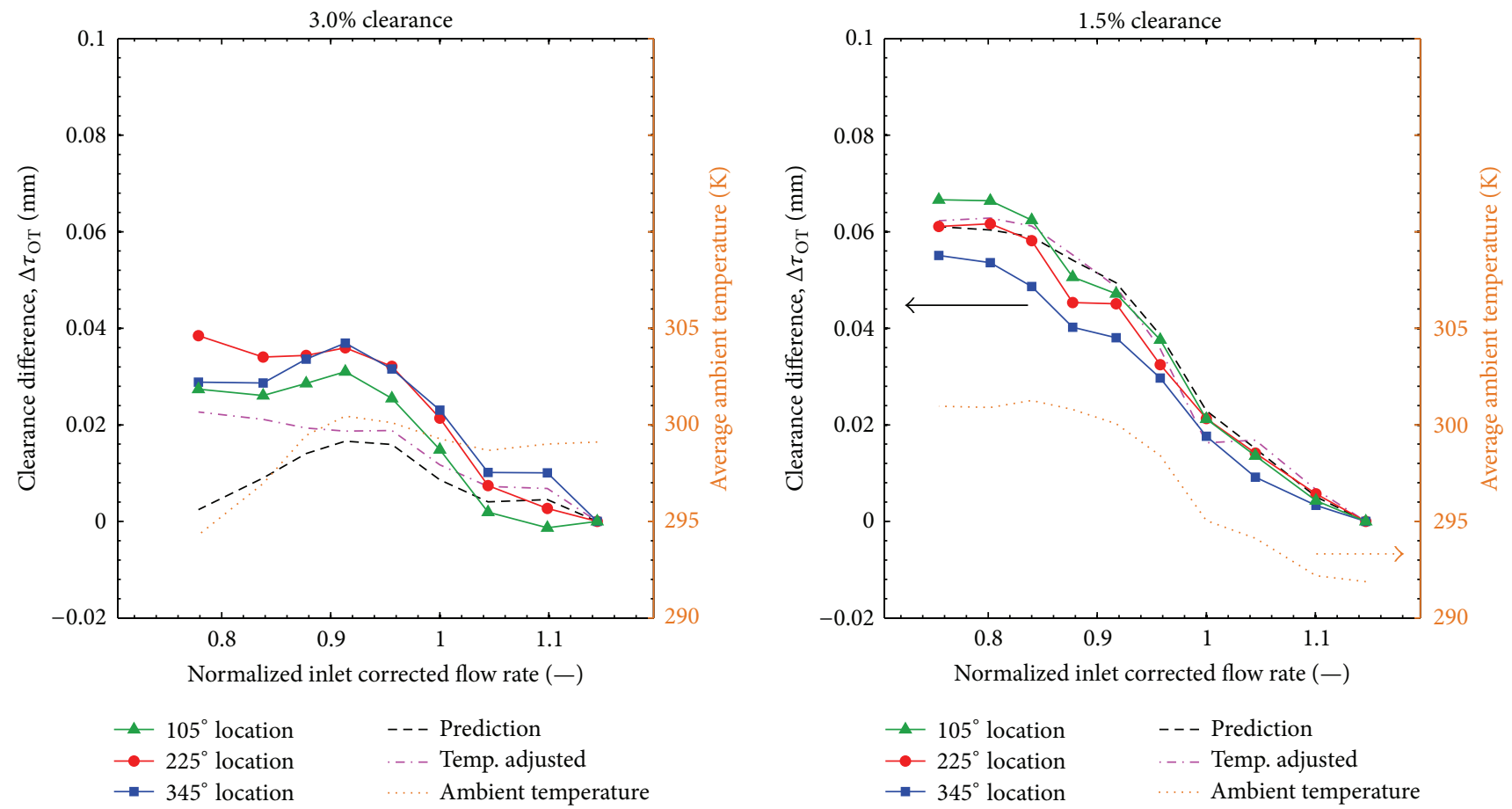

FIGURE 12: Predicted and measured clearance variations with loading condition, presented as a difference from the open throttle condition for different compressor tip clearance configurations with variations due to ambient conditions $\left(100 \% N_{c}\right.$, Rotor 1$)$.

temperatures measured at the outer diameter of the compressor shroud show no change. In this period (Region I; approximately 2-3 minutes), an increase of flow temperatures exists, but there is a definitive lag in the clearance response due to thermal growth. Over the course of the following 15 minutes (Region II), however, the temperature increase measured at the outer diameter of the compressor shroud is observable for all three rotors following an asymptotic trend. During this same period, the measured clearances also show an increase, but the trends for the three rotors differ slightly.

Throughout the entirety of Region II, the clearance change over Rotor 1 is approximately linear. In contrast, 


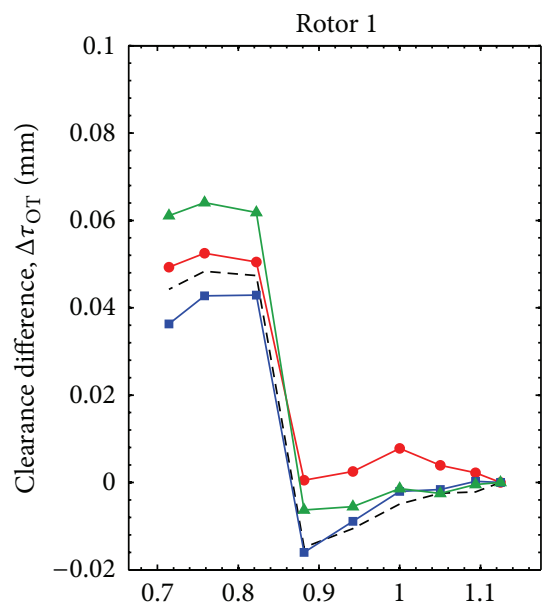

Normalized inlet corrected flow rate (-)

$\rightarrow 105^{\circ}$ location $\rightarrow-345^{\circ}$ location

$\rightarrow 225^{\circ}$ location - - - Prediction

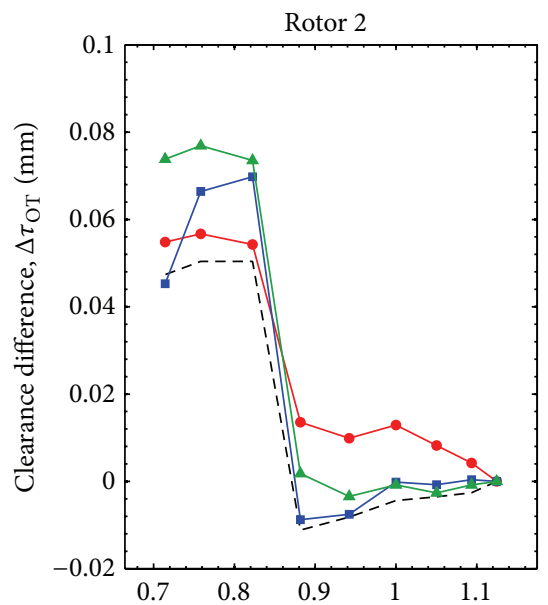

Normalized inlet corrected flow rate (-)

$\triangle 105^{\circ}$ location $\rightarrow 345^{\circ}$ location

$\rightarrow 225^{\circ}$ location - - - Prediction

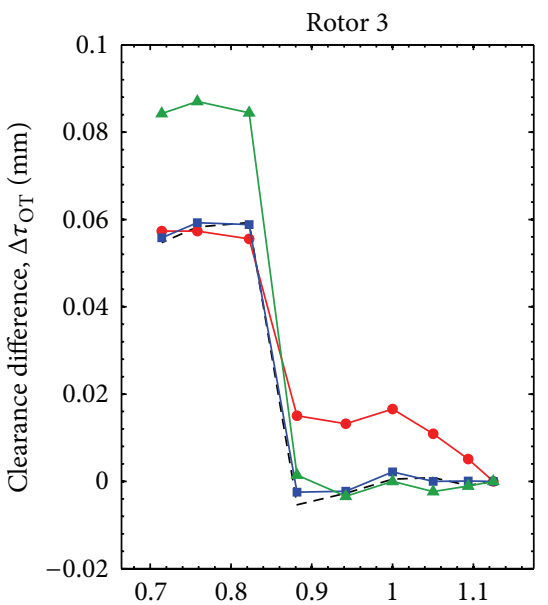

Normalized inlet corrected flow rate (-)

$\triangle 105^{\circ}$ location $\rightarrow 345^{\circ}$ location

- $225^{\circ}$ location - - Prediction

FIGURE 13: Predicted and measured clearance variations with loading condition, presented as a difference from the open throttle condition $\left(80 \% N_{c}\right)$ for compressor testing with a "stop and start" (1.5\% tip clearance).

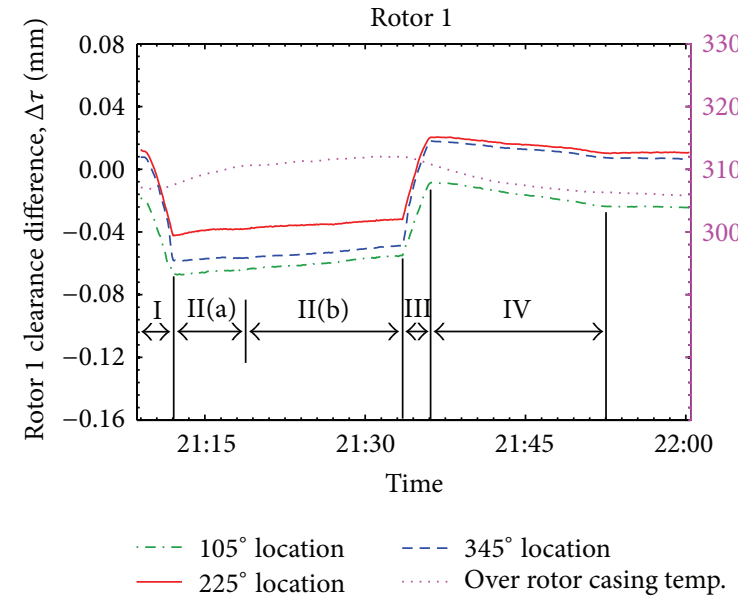

(a)

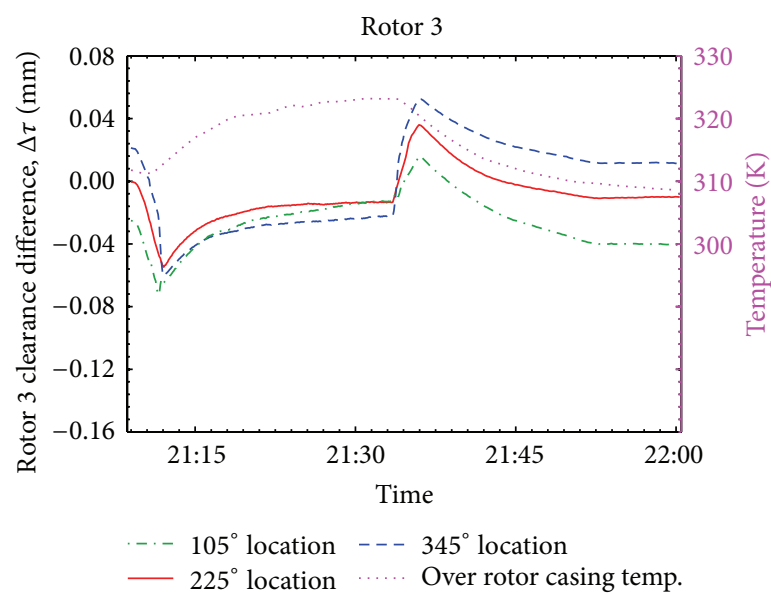

(c)

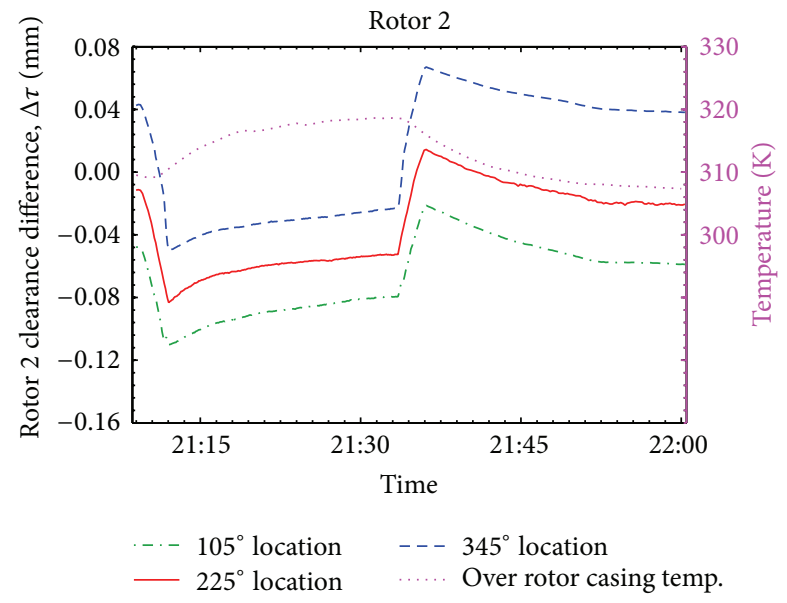

(b)

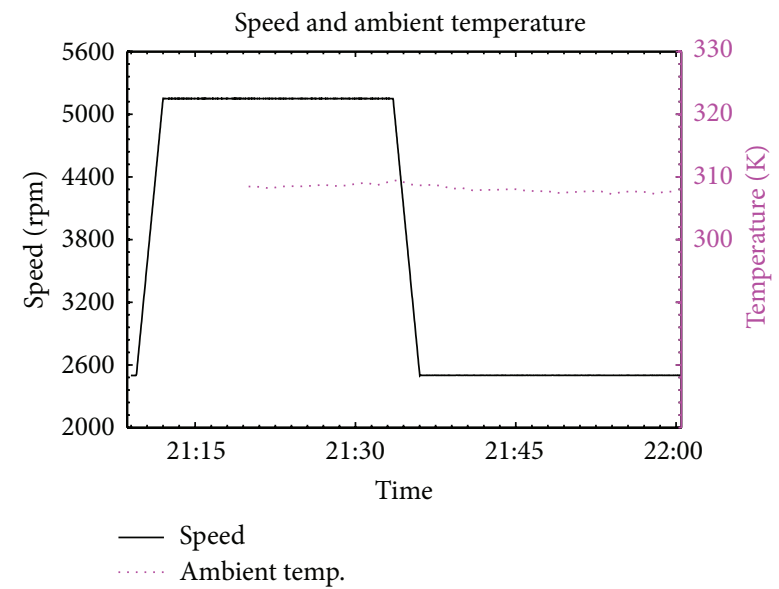

(d)

FIGURE 14: Clearance changes due to compressor speed changes and corresponding thermal variations at a nominal loading condition. 
the clearance changes over Rotor 2 and Rotor 3 exhibit a nonlinear section, Region II(a), which extends across approximately the initial one-third of Region II. The nonlinear response of Region $\mathrm{II}(\mathrm{a})$ is expectedly due to the more immediate change of tip clearance due to temperature rise in the flow (e.g., the contribution $u_{B \text {,thermal }}$ ). For this reason, the clearance change in Region II(a) for Rotor 3 is more significant than that of Rotor 2, and the relatively small temperature rise across Rotor 1 leads to a nondiscernable Region II(a) for that blade row. Region II(b), on the other hand, is primarily a contribution due to the heat transfer through the compressor shroud contributing to the thermal growth term $u_{S \text {,thermal }}$.

Once the measured temperatures at the outer diameter of the shroud approached an asymptotic value, the rotational speed was decreased at the same rate of $1100 \mathrm{rpm}$ per minute. Predictably, the trends of measured clearances show a sharp increase with the decreased rotational speed as the clearance contributions due to centrifugal forces respond (Region III). In contrast with Region II, the thermally dominated Region IV for the deceleration follows a more linear trend for all three rotors. This important difference is related to the cooling process imposed by decreasing speed as the temperature rise through the compressor decreases and the convective heat transfer to the air around the compressor increases. As a result, there is a less distinct separation of thermal contributions in Region IV. Although there is a slight asymptotic trend in the clearance variations of Region IV for Rotor 3, the trend is significantly less apparent than it was for Region II with the same blade row.

\section{Summary and Conclusions}

Operating rotor tip clearances have been measured using an FM capacitance probe measurement system with the Purdue three-stage axial compressor. Due to the large amount of experimental data available, this facility provides a unique opportunity to examine tip clearance variations due to several external factors. In particular, tip clearance changes due to rotational speed, loading condition, ambient temperature, and thermal equilibrium have been addressed. This study has experimentally validated the existence of measurable changes in tip clearance at several loading conditions and for several different compressor tip clearance heights. Many of these measured changes are well beyond the calculated uncertainty of the electronics system, and nearly all are beyond the repeatability of the measurements.

The results presented herein pose a very real problem for operating gas turbine engines that has not been presented previously through experimental data. Previous authors have discussed the potential variation of rotor tip clearance with changing loading condition, but this changing clearance is typically not implemented in computational models. These measurable changes of tip clearance with operating condition could be important for comparing experimental and computational performance. More importantly, these measured clearance changes with ambient operating conditions can also lead to measurable changes in overall total pressure ratio beyond the uncertainty of the measurements.
These results have focused on a machine which yields relatively low total temperature ratios. Thus, the thermally driven clearance changes could be considered small in comparison with larger multistage compressors. In particular, it has been shown that the largest clearance variations exist in the rear stages where the temperature rise is the greatest. For typical gas turbine compressor designs, this is the location at which the blade heights and the relative tip clearances are the smallest. As a result, the change of tip clearance with operating condition and ambient temperature is likely more significant than some may believe.

Ultimately, the results presented here motivate a need to incorporate tip clearance measurement systems with compressor test facilities desiring to accurately monitor compressor performance. Experimental data collected at different times of day, during different periods of the calendar year, or at different loading conditions can lead to significant variations of tip clearances which must be monitored for a best comparison with computational tools.

\section{Nomenclature}

FLA: Forward looking aft

$\tau$ : Operating tip clearance height

$\tau_{\mathrm{OT}}$ : Operating tip clearance height at open throttle

$\tau_{\text {static }}:$ Static tip clearance height

$u_{B}$ : Clearance contribution due to blade deformation

$u_{D}$ : Clearance contribution due to disc deformation

$u_{S}: \quad$ Clearance contribution due to shroud deformation.

\section{Conflict of Interests}

The authors declare that there is no conflict of interests regarding the publication of this paper.

\section{Acknowledgments}

This material is based upon work supported by NASA under the ROA-2010 NRA of the Subsonic Fixed Wing project and in part by the National Science Foundation Graduate Research Fellowship Program under Grant no. DGE-1333468. The authors are grateful to Reg Morton for providing technical information specific to the probe measurement system. The authors would also like to thank Rolls-Royce for the permission to publish this work.

\section{References}

[1] D. C. Wisler, "Loss reduction in axial-flow compressors through low-speed model testing," ASME Journal of Engineering for Gas Turbines and Power, vol. 107, no. 2, pp. 354-363, 1985.

[2] C. C. Koch and L. H. Smith Jr., "Loss sources and magnitudes in axial-flow compressors," ASME Journal of Engineering for Gas Turbines and Power, vol. 98, no. 3, pp. 411-424, 1976.

[3] J. P. Barranger, "Recent advances in capacitance type of blade tip clearance measurements," NASA Technical Memorandum 101291, 1998.

[4] J. D. Denton, "Some limitations of turbomachinery CFD," ASME Paper GT2010-22540, American Society of Mechanical Engineers, 2010. 
[5] P. P. Walsh and P. Fletcher, Gas Turbine Performance, Blackwell Science, Oxford, UK, 2008.

[6] N. R. Smith, R. A. Berdanier, J. C. Fabian, and N. L. Key, "Reconciling compressor performance differences with varying ambient conditions," ASME Paper POWER2015-49102, American Society of Mechanical Engineers, 2015.

[7] D. E. van Zante, A. J. Strazisar, J. R. Wood, M. D. Hathaway, and T. H. Okiishi, "Recommendations for achieving accurate numerical simulation of tip clearance flows in transonic compressor rotors," ASME Journal of Turbomachinery, vol. 122, no. 4, pp. 733-742, 2000.

[8] A. G. Sheard, "Blade by blade tip clearance measurement," International Journal of Rotating Machinery, vol. 2011, Article ID 516128, 13 pages, 2011.

[9] J. Chivers, "A technique for the measurement of blade tip clearance in a gas turbine," AIAA Paper 89-2916, AIAA, 1989.

[10] H. Agarwal, S. Akkaram, S. Shetye, and A. McCallum, "Reduced order clearance models for gas turbine applications," AIAA Paper 2008-2177, AIAA, 2008.

[11] J. A. Kypuros and K. J. Melcher, "A reduced model for prediction of thermal and rotational effects on turbine tip clearance," NASA Technical Memorandum 2003-212226, 2003.

[12] Y. Dong, Z. Xinqian, and L. Qiushi, "An 11-stage axial compressor performance simulation considering the change of tip clearance in different operating conditions," Proceedings of the Institution of Mechanical Engineers Part A: Journal of Power and Energy, vol. 228, no. 6, pp. 614-625, 2014.

[13] D. C. Ye, F. J. Duan, H. T. Guo, Y. Li, and K. Wang, “Turbine blade tip clearance measurement using a skewed dual-beam fiber optic sensor," Optical Engineering, vol. 51, no. 8, Article ID 081514, 2012.

[14] N. L. Key, "Influence of upstream and downstream compressor stators on rotor exit flow field," International Journal of Rotating Machinery, vol. 2014, Article ID 392352, 10 pages, 2014.

[15] T. N. Satish, R. Murthy, and A. K. Singh, "Analysis of uncertainties in measurement of rotor blade tip clearance in gas turbine engine under dynamic condition," Proceedings of the Institution of Mechanical Engineers, Part G: Journal of Aerospace Engineering, vol. 228, no. 5, pp. 652-670, 2014.

[16] D. Müller, A. G. Sheard, S. Mozumdar, and E. Johann, "Capacitive measurement of compressor and turbine blade tip to casing running clearance," ASME Journal of Engineering for Gas Turbines and Power, vol. 119, no. 4, pp. 877-884, 1997.

[17] J. R. Brossman, An investigation of rotor tip leakage flows in the rear-block of a multistage compressor [Ph.D. thesis], Purdue University, West Lafayette, Ind, USA, 2012.

[18] E. W. Lemmon, M. L. Huber, and M. O. McLinden, NIST Standard Reference Database 23: Reference Fluid Thermodynamic and Transport Properties-REFPROP, Version 9.1, National Institute of Standards and Technology, Standard Reference Data Program, Gaithersburg, Md, USA, 2013.

[19] L. K. Baxter, Capacitive Sensors: Design and Applications, WileyIEEE Press, Hoboken, NJ, USA, 1996. 

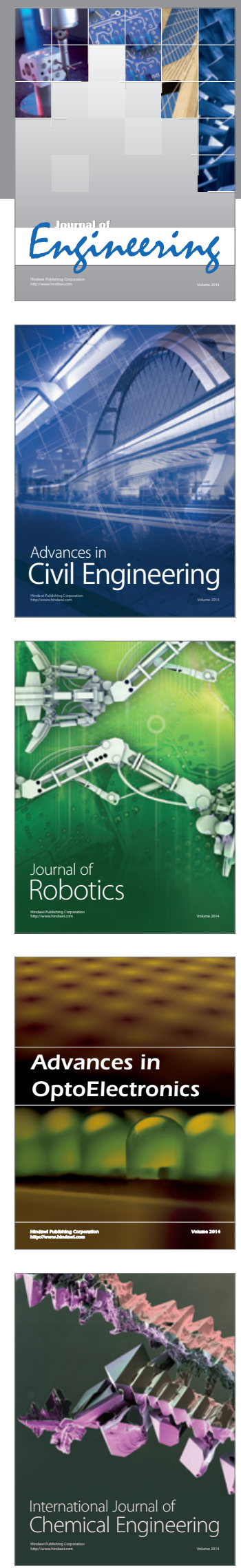

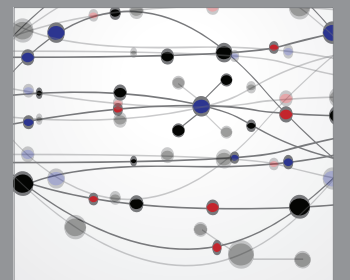

The Scientific World Journal
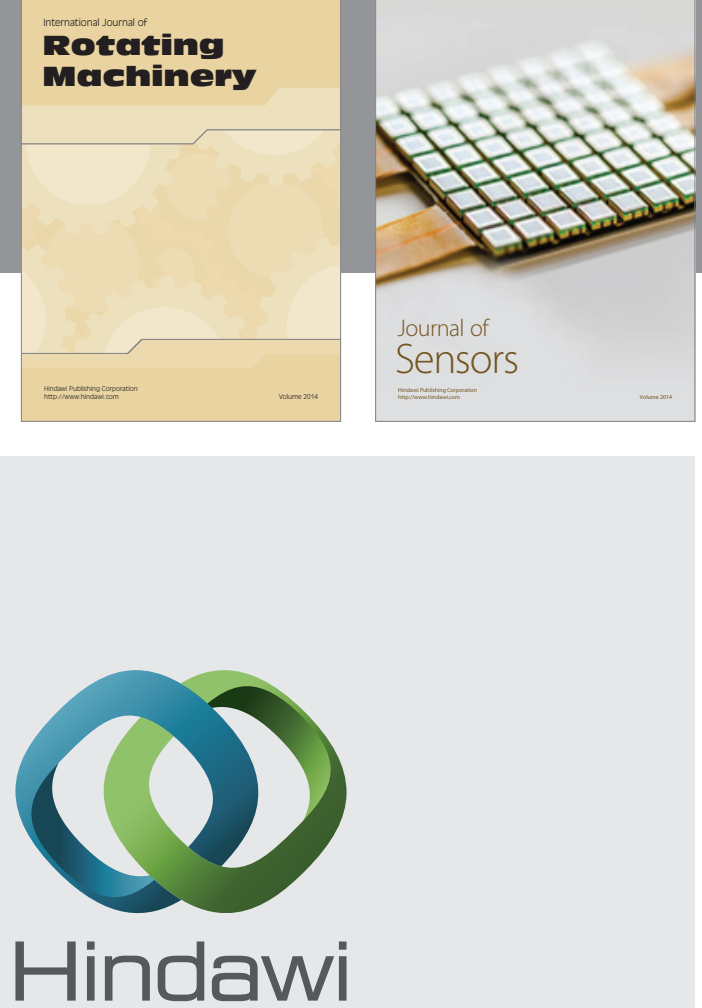

Submit your manuscripts at http://www.hindawi.com
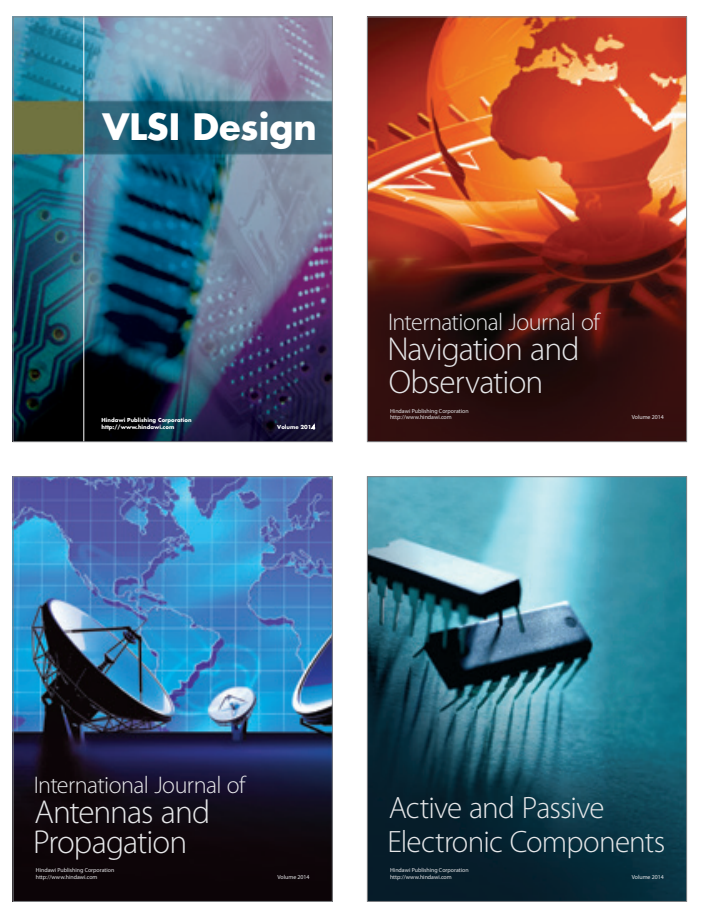
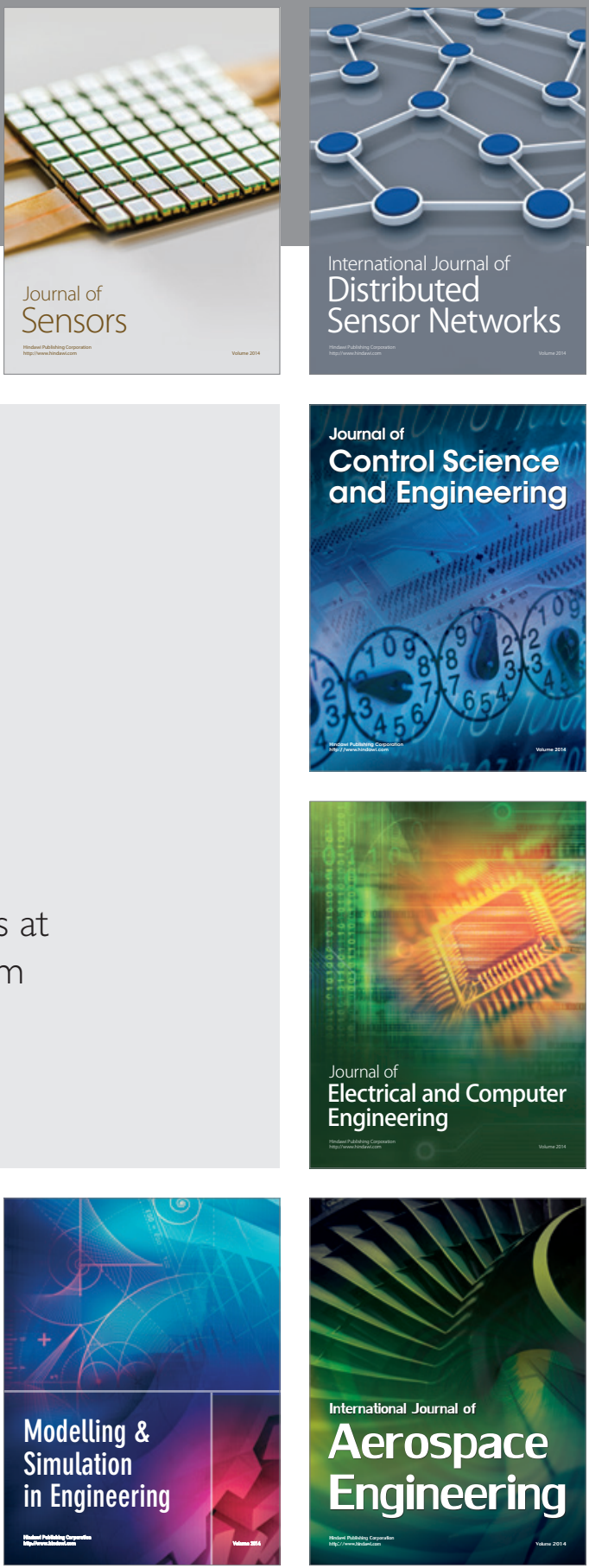

Journal of

Control Science

and Engineering
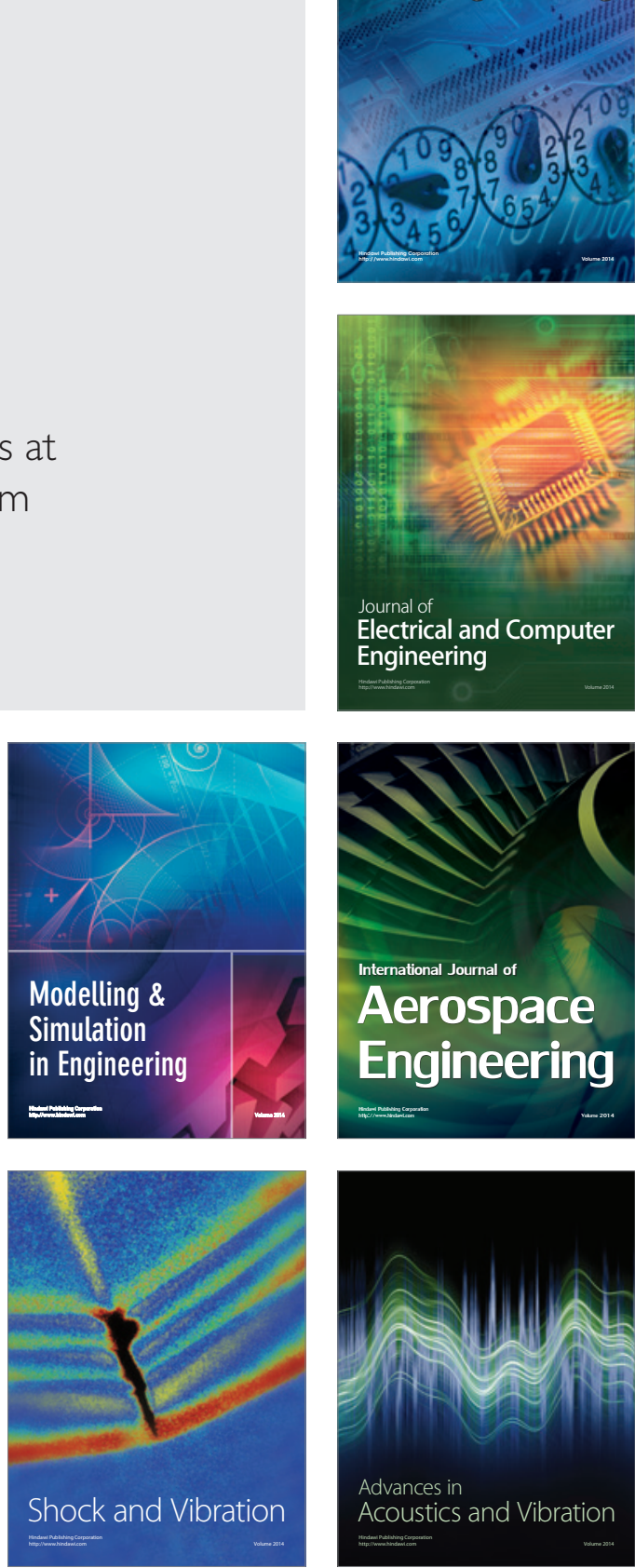